\title{
Erzählverfahren und Erzähltechniken dokufiktionalen Erzählens
}

Wenn man dokufiktionales Erzählen in der Literatur nicht als ein Genre, sondern als eine „Schreibweise“1 oder einen Darstellungsmodus auffasst, der „in unterschiedlichen Genres anzutreffen ist" ${ }^{\text {, }}$, verliert die Frage, ob der Text nun als Ganzer der Fiktion oder dem sogenannten faktualen Erzählen zuzuordnen ist, an Bedeutung. ${ }^{3}$ Kennzeichnend für diesen Darstellungsmodus sei, so Agnes Bidmon, dass die Texte auf der Ebene des discours ,dokumentarische und fiktionale Darstellungsweisen mithilfe intertextueller, intermedialer oder transmedialer Verfahren amalgamieren“. ${ }^{4}$ An anderer Stelle heißt es, für die „grundlegenden ästhetischen wie ethischen Maximen des dokumentarischen Erzählens“ spiele es eine Rolle, „dass die im dokumentarischen Material eingefangenen Spuren von Realität explizit sichtbar gemacht werden“; dies geschehe dadurch, dass dieses Material durch verschiedene Verfahren „in den Text einmontiert“ werde. ${ }^{5}$ Zwischen Einmontieren (wo die Heterogenität sichtbar bleibt) und Amalgamieren (wo sie nur noch dem Analysierenden zugänglich ist) bewegen sich die dokufiktionalen Erzählverfahren und Erzähltechniken. Damit geht es letztlich auch um die Frage, in welcher Weise sich das Dokumentarische dokumentiert. Weil es sich dokumentiert, befriedigt dokufiktionales Erzählen keinen so genannten ,Wirklichkeitshun-

1 Bidmon, Agnes. „Streng vertraulich! Dokufiktionales Erzählen als Schreibweise des Politischen in der Literatur der Gegenwart anhand von Ilija Trojanows Macht und Widerstand“. In: Politische Literatur. Begriffe, Debatten, Aktualität. Hg. Christine Lubkoll, Manuel Illi, Anna Hampel. Stuttgart: J. B. Metzler 2019, 421-440.

2 Bidmon: „Streng vertraulich!“, 429.

3 Neuere Fiktionalitätstheorien präferieren eine pragmatische Definition von Fiktionalität. Christian Klein und Matías Martínez fassen lapidar zusammen: „Die Klassifikation eines Textes als fiktional oder faktual ist eine Entscheidung, die letztlich auf textpragmatischer Ebene getroffen wird.“ (Klein, Christian/Martínez, Matías. „Wirklichkeitserzählungen. Felder, Formen und Funktionen nicht-literarischen Erzählens“. In: Wirklichkeitserzählungen. Felder, Formen und Funktionen nicht-literarischen Erzählens. Hg. Christian Klein, Matías Martínez. Stuttgart: J. B. Metzler 2009, 1-13, hier 4.) Man kann aber auch bestreiten, dass es - pragmatisch gesehen einen Zwang gibt, diese pragmatische Entscheidung zu treffen. Für den vorliegenden Zusammenhang, der sich nicht mit ,ganzen Texten“ beschäftigt, kann diese Frage zurückgestellt werden.

4 Bidmon: „Streng vertraulich!“, 429.

5 Bidmon: „Streng vertraulich!“, 429. 
ger"6. Aber es kann als eine Antwort auf einen solchen,Wirklichkeitshunger` verstanden werden.

Dokufiktion wäre also die Einheit der Differenz von Dokument und Fiktion, weil der Unterschied von Fiktion und Dokument im dokufiktionalen Text irgendwie vorkommen, d.h. markiert sein muss. Dadurch wird die Vorstellung verhindert, dass er auf eine ,Wirklichkeit‘ hin transparent sei. Weder gibt ein Dokument eine objektive oder subjektive Wirklichkeit wieder, wie es ein autobiographischer Pakt oder ein „Faktualitätspakt“7 suggeriert, noch ist die Fiktion eine fiktionale Wirklichkeit (wie es die merkwürdige Rede vom Fiktionsvertrag nahelegt ${ }^{8}$ ). Insofern ist der dokufiktionale Text etwas, was sich nicht selbst genügt.

Um genauer zu verstehen, wie Dokument und Fiktion in einem Text ineinandergreifen und sich aufeinander beziehen können, muss man verschiedene Grade oder Modi des Dokumentarischen und des Fiktionalen unterscheiden. Was das Dokumentarische angeht, so bekommen wir in einem literarischen Text natürlich niemals ein Dokument zu Gesicht, sondern immer nur die Wiedergabe eines Dokuments. Diese Wiedergabe kann mehr oder weniger vollständig sein: als Kopie eines sprechenden Dokuments wie etwa eines Reisepasses wie in W. G. Sebalds Schwindel. Gefühle, ${ }^{9}$ als stumme Fotografie oder sonstiges Bildzitat (mit oder ohne Legende), als typographisch hervorgehobenes Schriftstück wie in Ein unsicht-

\footnotetext{
6 Vgl. zum Stand der Diskussion etwa Gittel, Benjamin. „,Wirklichkeitsverlust', ,Wirklichkeitshunger‘ und ,Neuer Realismus‘. Zur Verschränkung von Gegenwartsdiagnostik, Poetologie und Literaturwissenschaft“. In: IASL 43/1 (2018), 68-89.

7 Martínez, Matías. „Erzählen im Journalismus“. In: Wirklichkeitserzählungen. Felder, Formen und Funktionen nicht-literarischen Erzählens. Hg. Christian Klein, Matías Martínez. Stuttgart: J. B. Metzler 2009, 179-191, hier 185.

8 Die Rede vom Fiktionsvertrag (als Gegenstück zum autobiografischen Pakt à la Lejeune) wird häufig mit der berühmten Formel von Coleridge als ,willentliche Aussetzung des Unglaubens‘ (willing suspension of disbelief) aufgefasst. So schreibt z. B. Jochen Mecke: „Die Produktion und Rezeption von Literatur vollzieht sich im kommunikativen Rahmen eines ,Paktes der Fiktion', der alle drei literarischen Gattungen umfasst und eine ,suspension of disbelief“ voraussetzt, also die freiwillige Außer-Kraft-Setzung des Misstrauens seitens des Lesers.“ (Mecke, Jochen. „Du musst dran glauben. Von der Literatur der Lüge zur Lüge der Literatur“. In: Diegesis 4/1 (2015). https://www.diegesis.uni-wuppertal.de/index.php/diegesis/article/view/188/255 (28.10.2019)). Dem Autor wird in einem solchen Fiktionsvertrag also, vereinfacht gesprochen, die Lizenz zum Lügen erteilt, weshalb die Lüge dann keine ist. Das ist ein seltsamer Vertrag, da er dem Autor alle Rechte einräumt und ihn zu nichts verpflichtet. Von einem Vertrag kann man sinnvoll eigentlich nur sprechen, wenn dem Leser auf diese Weise die Frage, ob er nun etwas glauben soll oder nicht, dadurch abgenommen wird. Aber so funktioniert das eben höchstens bei Märchen und Verwandtem.
}

9 Sebald, W. G. Schwindel. Gefühle. Frankfurt a. M.: Fischer 1994, 135. 
bares Land von Stephan Wackwitz, ${ }^{10}$ als ausgewiesenes oder unausgewiesenes wörtliches Zitat, als sinngemäße Wiedergabe mit eingebundenen Zitaten.

Was die Grade und Modi des Fiktionalen angeht, denen die folgenden Überlegungen gewidmet sind, so kennt die klassische Lehre von den sogenannten Fiktionssignalen keine Abstufung. Wenn aber, wie ich eingangs behauptet habe, die Einordnung eines Textganzen als fiktional oder faktual aus der Perspektive des Dokufiktionalen an Bedeutung verliert, muss auch dies überdacht werden. Entsprechend weiß man in der neueren Theorie, dass der Begriff des (textinternen ${ }^{11}$ ) Fiktionssignals insofern problematisch ist, als das Wort Signal eine absichtliche Kundgabe unterstellt, während die Zeichen, die Fiktionalität gegebenenfalls anzeigen, unter Umständen auch als Symptome oder Indizien klassifiziert werden können. ${ }^{12}$

Die Zone der Unbestimmtheit, die durch diese Erwägung für das dokufiktionale Erzählen eröffnet wird, soll im Folgenden tentativ an vier Beispielen beleuchtet werden, in denen es einerseits eine Bindung an das Dokumentarische gibt und andererseits eine Überschreitung des Dokumentarischen - was die Frage nach einem etwaigen „semidokumentarischen Pakt"13 aufwirft. In allen vier Beispielen geht es um die Ebene der Darstellung: Daher taucht z. B. in keinem der Beispiele eine fiktive Figur auf.

\section{1.}

Ich beginne mit einem scheinbaren dokufiktionalen Nullpunkt: 1913. Der Sommer des Jahrhunderts von Florian Illies aus dem Jahre 2012. Bekanntlich ist das Buch, mit den Monaten als Kapiteln, wie eine Chronik angelegt und wirft Schlaglichter auf eine größere Anzahl vor allem deutscher Geistes- bzw. Kulturgrößen am Vorabend des ungeahnten Ersten Weltkriegs. Die Schlaglichter sind mal nur einen Satz lang - z. B.: „Apropos kränkelnd: Wo steckt eigentlich Rilke?“14 -, mal auch mehrere Seiten.

10 Wackwitz, Stephan. Ein unsichtbares Land. Familienroman. Frankfurt a. M.: Fischer 2005. 11 Textexterne, also peritextuelle und epitextuelle Fiktionssignale, sind für den vorliegenden Problemzusammenhang nicht relevant, da sie sich per definitionem nur auf den ganzen Text beziehen.

12 Zipfel, Frank. „Fiktionssignale“. In: Fiktionalität. Ein interdisziplinäres Handbuch. Hg. Tobias Klauk, Tilmann Köppe. Berlin/Boston: De Gruyter 2014, 97-124, hier 103.

13 Bidmon: „Streng vertraulich!“, 430.

14 Illies, Florian. 1913. Der Sommer des Jahrhunderts. Frankfurt a. M.: Fischer 2014, 13. 
Eines der bei diesen Schlaglichtern zum Einsatz kommenden Selektionsprinzipien ist die Dokumentierbarkeit: Was nicht dokumentierbar ist, das kann auch nicht vorkommen. Insofern sind wir hier tatsächlich am Nullpunkt des Fiktionalen. Das Buch von Illies war längere Zeit auf Platz 1 der Spiegel-Bestsellerliste im Bereich Sachbuch und nimmt entsprechend keinerlei Fiktionalität (etwa im Paratext) für sich in Anspruch. Der Bestsellerrang wurde dem Buch aber nur zuteil, weil gleichwohl dokufiktionale Verfahren angewandt worden waren. In der folgenden Passage hat Else Lasker-Schüler in ihrem klirrend kalten Mansardenzimmer eine unverhoffte Postkarte von Franz Marc bekommen:

Sie erstarrt in stillem Jubel. Auf winzigem Raum hat ihr ferner Freund hier einen ,Turm der blauen Pferde' gemalt, kraftstrotzende Tiere, die sich zum Himmel türmen, ganz aus der Zeit gefallen und doch in ihr stehend. Sie spürt, dass sie ein einzigartiges Geschenk bekommen hat: die ersten blauen Pferde des Blauen Reiters. Vielleicht spürt diese besondere Frau, die immer alles spürt, sogar noch mehr - dass aus der Idee dieser Postkarte in den Wochen danach im fernen Sindelsdorf ein noch viel größerer ,Turm der blauen Pferde‘ entstehen sollte, ein Gemälde als Programm, ein Jahrhundertbild. Es wird später verbrennen, und es wird allein diese Postkarte sein, die Fingerabdrücke von Franz Marc und Else Lasker-Schüler bis heute bewahrt hat, die auf alle Ewigkeit von dem Moment erzählen wird, als der Blaue Reiter zu galoppieren begann. ${ }^{15}$

Man kann hier das Zusammenspiel einiger Elemente beobachten. Einerseits wird eine Szene aufgerufen, in der eine bekannte historische Figur allein (also ohne Zeugen) ist. Darüber hinaus werden aber auch Aussagen über ,innere Zustände، dieser Figur gemacht, von denen zunächst einmal auch und gerade die Erzählinstanz nichts wissen kann („Sie erstarrt in stillem Jubel“; „Sie spürt [...]“). Das heißt aber nicht, dass die Mitteilungen dieser inneren Zustände als Fiktionssignale fungieren. Sie müssen auch keineswegs als Symptome oder Indizien für das Vorliegen von Fiktionalität gewertet werden. Denn es ist möglich, dass sie über sogenannte Ego-Dokumente wie insbesondere Briefe belegt werden können. ${ }^{16}$ Diese Dokumentierbarkeit liegt nicht zuletzt deshalb nahe, weil es sich um einen zwar intimen, zugleich aber herausgehobenen Moment handelt, der, wie der zitierte Abschnitt vorführt, verschiedene Bezüge und Anschlussmöglichkeiten bietet. Diese Dokumentierbarkeit geht strukturell gesehen bereits in die Rezeptionssituation ein: Wir unterstellen aus verschiedenen Gründen, dass der Erzähler bzw. Illies das nicht schreiben würde, wenn er es nicht belegen könnte.

15 Illies: 1913, 23.

16 In der dem Buch von Illies angehängten „Auswahlbibliographie“ ist Else Lasker-Schüler nicht aufgeführt, sondern nur die Biografie von Sigrid Bauschinger. Dort wird allerdings auf diese Szene Bezug genommen und aus einem Brief zitiert (vgl. Bauschinger, Sigrid. Else Lasker-Schüler. Eine Biographie. Göttingen: Wallstein 2004, 196-197). 
Und das wird ebenfalls in dieser Passage markiert, wenn es zunächst heißt, dass Else Lasker-Schüler etwas ,spürt', dann aber, dass sie, vielleicht' darüber hinaus noch mehr ,spürt‘. Damit deutet der Erzähler ja an, dass er in der aufgerufenen Situation eben keinen unmittelbaren Zugang zu den inneren Zuständen der Figur hat und daher auf Vermutungen angewiesen ist. Die Formen des vermutenden oder konjekturalen Erzählens sind - wie noch genauer gezeigt werden soll - ein zentrales Verfahren des Dokufiktionalen. Das Konjekturale impliziert wiederum, dass der Erzähler sowohl eine Instanz ist als auch eine subjektive Spur hinterlässt: Er sagt (als Instanz) zwar nicht mehr, als er sich zu vermuten berechtigt sieht, aber zugleich schlägt sich darin das subjektive Begehren nieder, mehr darüber zu wissen. Dies wird zusätzlich signalisiert durch die Wahl des Präsens als Darstellungsmodus. Das narrative Präsens kann gewiss sehr verschiedene Funktionen erfüllen, ${ }^{17}$ hier aber markiert es zunächst ganz klassisch statt der ,erzählten Welt ${ }^{\text {‘ }}$ die ,besprochene Welt',18 in der eine andere Position des Sprechers impliziert ist. Die Subjektposition des dokufiktionalen Erzählers ist strukturell durch ein Begehren nach Dokumentierbarkeit gekennzeichnet.

Entsprechend - das ist ein weiteres Element - kann dieses Subjekt das vermutete ,Spüren“ der Figur proleptisch mit einer Zukunft verbinden und die erzählte Situation auf diese Weise auratisieren. Franz Marcs gleichnamiges großformatiges „Jahrhundertgemälde“ ist verschollen, ${ }^{19}$ doch das in 1913. Der Sommer des Jahrhunderts zwar nicht gezeigte, aber im Prinzip vorzeigbare Dokument, die Postkarte $\mathrm{e}^{20}$, bewahrt, wie erzählt wird, noch die Fingerabdrücke von Sender und Adressat usw. Darin liegt natürlich auch eine zusätzliche Rechtfertigung dafür, von diesem Moment zu erzählen (schon die Postkarte selbst ,erzählt', d. h. fordert zu einem das Dokumentarische erweiternden Erzählen auf).

Was der Erzähler hier dem Wissenshorizont von Else Lasker-Schüler in der konkreten Situation aus eigenem - hier späterem - Wissen hinzufügt, kommt einer thematischen Verlagerung gleich. Eine Erzählung kann sich - gleichsam unter einem Vorwand - jederzeit von der entworfenen Situation in einer zeitweiligen Abschweifung entfernen.21 Über dieses Verfahren kann etwas nicht Dokumen-

17 Vgl. für einen Überblick den Sammelband Avanessian, Armen/Hennig, Anke (Hg.). Der Präsensroman. Berlin/New York: De Gruyter 2013. Anders als der Titel vermuten lässt, geht es in den Beiträgen dieses Bandes nicht nur um den Roman.

18 Weinrich, Harald. Tempus. Besprochene und erzählte Welt. München: C.H.Beck 2001 [1964].

19 Es befand sich zuletzt im Besitz von Hermann Göring und es ist keineswegs sicher, dass es verbrannt ist.

20 Sie befindet sich in der Staatlichen Graphischen Sammlung in München.

21 Im vorliegenden Fall fährt die Erzählung im nächsten Absatz mit den Worten „Gerührt sieht die Dichterin, wie der große Maler [...]“ (Illies: 1913, 23) fort. 
tiertes durch etwas Dokumentierbares ersetzt werden, das die konkrete Situation überschreitet und im Modus einer größeren Distanz wiedergegeben wird. An die Stelle der nicht möglichen internen Fokalisierung tritt dann eine Nullfokalisierung, die gerade nicht mit einem ,allwissenden Erzähler‘ gleichzusetzen ist. Dies ist, da ja die ,Schlaglichter' die Dokumentierbarkeit zur Voraussetzung haben, das allgemeinste dokufiktionale Verfahren in 1913. Es handelt sich um ein Verfahren, das nur bei historischen Personen mit gut dokumentiertem Leben möglich ist (und darin liegt vielleicht auch das Ärgerliche dieses Buches).

\section{2.}

Mein zweites Beispiel ist ebenfalls ein sogenanntes Sachbuch. Es heißt Die Staatsräte. Elite im Dritten Reich: Gründgens, Furtwängler, Sauerbruch, Schmitt, ist aus dem Jahr 2018 und stammt aus der Feder des Literatur- und Kulturwissenschaftlers Helmut Lethen. Das Thema des Buches geht aus dem Titel hervor. 1933 wurde unter der Leitung von Hermann Göring mit großem Pomp der Preußische Staatsrat ins Leben gerufen, mit dem der NS-Staat einflussreiche Intellektuelle an sich binden wollte. Die vier genannten Personen gehörten diesem Staatsrat an (der im Übrigen nur wenige Male einberufen wurde). Diese Verhältnisse werden im ersten Teil des Buches unter Einbeziehung von konkret nachgewiesenen Quellen, Forschungsliteratur usw. eingehend geschildert und analysiert.

Der Hauptteil des Buches ist jedoch mit „Geistergespräche“ (erster und zweiter Teil) überschrieben. ${ }^{22}$ In diesen Geistergesprächen trifft sich das Quartett der Theatermann, der Dirigent, der Starchirurg und der Staatsrechtler -, unterhält sich und diskutiert, obwohl Helmut Lethen in der Einleitung erklärt, er habe „kein Dokument finden“ können, „das bezeugt hätte, dass sich Gründgens, Furtwängler, Sauerbruch und Schmitt jemals getroffen haben. Umso reizvoller war es, ihre Treffen zu erfinden. An Originaltönen herrscht kein Mangel, aber die Fiktion spricht lauter." 23 Weil Lethen ein Literaturwissenschaftler ist, benennt er in der Danksagung am Ende auch noch einmal korrekt, was er gemacht hat. Er habe nämlich die Arbeit an diesem Buch für einige Zeit liegen lassen müssen, weil er das Gefühl gehabt habe, „die Vorstellung dieses nicht existierenden ,Quartetts“ höchstens in Form eines Puppenspiels realisieren zu können.“ Eine „Dokufikti-

22 Lethen, Helmut. Die Staatsräte. Elite im Dritten Reich: Gründgens, Furtwängler, Sauerbruch, Schmitt. Berlin: Rowohlt 2018, 85-200 und 273-298.

23 Lethen: Die Staatsräte, 16. 
on“, so Lethen weiter, müsse aber „eine Masse an Quellen, Wortlauten und Gesten verschmelzen [...], wozu eigentlich nur ein Romancier fähig“ sei. ${ }^{24}$

Wie bereits diese Kommentare deutlich machen, geht es hier um eine ausgestellte Fiktionalität. Der fiktionale Anteil wird kategorial von einem nichtfiktionalen Teil gerahmt und abgegrenzt und dadurch legitimiert. Insofern handelt es sich um einen hybriden Text. ${ }^{25}$ Das heißt beim dokufiktionalen Erzählen aber gerade nicht, dass innerhalb dieses Bereichs eine Art ,Narrenfreiheit“ des ungebundenen Fabulierens herrscht. Gerade innerhalb der Fiktion muss ja die „Masse an Quellen“ verschmolzen, also amalgamiert werden. Die Schwierigkeiten einer solchen Verschmelzung werden hier in die Metapher des Puppenspiels gefasst: Die vier illustren Herren drohen bloße Puppen zu werden, weil sie insbesondere in ihren übrigens nie mit Zitatzeichen versehenen - Reden und Bemerkungen Dokumentiertes zum Besten geben. Ich gebe, um das Verfahren zu veranschaulichen, ein sowohl einfaches wie auch etwas perfides Beispiel. Im dritten Geistergespräch des Quartetts, abgehalten am Vorabend des Zweiten Weltkriegs, doziert der Chirurg Sauerbruch über Prothesen, und gegen Ende heißt es:

Gründgens wird die Unterhaltung zu sachlich, er kündigt eine Anekdote über „WiesengrundAdorno“ an. Schmitt und Furtwängler reagieren schon bei der Nennung des Namens leicht gereizt. Wiesengrund habe sie selbst erzählt, so Gründgens: die Geschichte von Adorno und der eisernen Prothesenhand. Er sei in einer Villa in Malibu, am Strand von Los Angeles, eingeladen gewesen. Einer der Gäste, als Hauptdarsteller des Films „The Best Years of Our Life“ berühmt, habe sich frühzeitig verabschiedet. Adorno habe ihm ein wenig geistesabwesend die Hand gegeben und sei dabei heftig zurückgezuckt. Der Abschiednehmende hatte im Krieg die Hand verloren und trug an deren statt [sic!] eine aus Eisen gefertigte, aber praktikable Klaue. Als die Eisenklaue den Druck der Hand erwiderte, habe Adorno sofort gespürt, dass er den Schrecken auf keinen Fall zeigen durfte. Also habe er versucht, das Schreckgesicht im Bruchteil einer Sekunde in eine verbindliche Miene zu verwandeln.

Gründgens führt den Schrecken pantomimisch vor und versaut damit die Pointe der Geschichte: Der ebenfalls eingeladene Chaplin - der Kriegsversehrte hatte inzwischen den Raum verlassen - habe zur großen Erbauung des Publikums und zur Beschämung des deutschen Philosophen die für Adorno peinliche Situation nachgespielt.

24 Lethen: Die Staatsräte, 349-350.

25 Christian Klein und Matías Martínez stellen fest, dass „Wirklichkeitserzählungen häufig hybride Texte“ seien; auf der Ebene der histoire weisen sie in diesem Zusammenhang auf „fiktive Fallgeschichten“ im „wirtschaftlichen oder juristischen Diskurs“ hin: „Da diese fiktiven Elemente aber im Sinne der Praxisregulierung oder der Festlegung allgemeiner Strukturmerkmale mit Bezug auf die außersprachliche Wirklichkeit funktionalisiert werden, verstehen wir sie als Wirklichkeitserzählungen.“ (Klein/Martínez: „Wirklichkeitserzählungen“, 7). Bis zu einem gewissen Grad ist der Fall der „Geistergespräche“ analog, weil auch hier das Fiktive im Dienst einer Erkenntnis über die ,Wirklichkeit‘ steht. Jedoch ist dies letztlich ein sehr unklares Kriterium. 
Lächerlichkeit entstehe, meint Gründgens, wenn man sich selbst für den Brennpunkt des Interesses halte, aber von anderen bei einer peinlichen Reaktion beobachtet werde.

Für jeden Mann, entgegnet Schmitt, sei so etwas peinlich. Er sei erstaunt, dass Adorno den Mut besessen habe, die Selbstdemütigung in einer Erzählung preiszugeben. Ob der Frankfurter Philosoph diese Geschichte mit einem Kommentar versehen hat?

Wenn er sich recht erinnere, so Gründgens, habe Adorno gesagt, dass alles Grauen immer nah am Lachen sei und nur auf diese Weise als Rettendes erkannt werden könne.

Darüber muss nun erst Schmitt und dann die ganze Runde herzlich lachen. Diese Juden!26

Da das Quartett sich nie in dieser Form getroffen hat, ist natürlich die ganze Unterhaltung eine Fiktion. Aber es ist sozusagen eine möglichst sparsame, gleichsam asketische Fiktion - eine Fiktion, die sich auf das Notwendige beschränkt. Und das Notwendige besteht in dem, was jeweils gesagt wird und wie die anderen auf dieses Gesagte reagieren. Die längeren Auslassungen sind dabei mehr oder weniger aus Schriften dokumentierbar (wenn etwa Carl Schmitt über den Begriff des Feindes spricht usw.), die möglichen Reaktionen sind hingegen vorgestellt.

Da die Geistergespräche als fiktional ausgewiesen sind, rücken sie tendenziell in die Nähe von Gedankenexperimenten: Wenn diese vier Staatsräte sich wirklich getroffen hätten, wie hätten sie in ihren Gesprächen aufeinander reagiert? Dieses Verfahren des hypothetischen Erzählens, wie man das nennen könnte, weist obzwar auf einer anderen Ebene - eine Verwandtschaft zum mutmaßenden oder konjekturalen Erzählen auf (das formal gesehen durch die Markierung als Fiktion innerhalb der Fiktion nicht mehr markiert werden kann), insofern es ebenfalls nicht in einfacher Weise etwas behauptet. $\mathrm{Zu}$ diesem Status passt, dass auch die Geistergespräche nicht im epischen Präteritum, sondern im Präsens erzählt sind, dieses Präsens aber eben nicht mehr auf eine besprochene, sondern auf eine zu Erkenntniszwecken konstruierte Welt verweist.

Das Besondere bzw. Perfide an diesem Ausschnitt hängt damit zusammen, dass Gründgens (ausnahmsweise) nicht etwas aus seinem eigenen Gedankengut in den Mund gelegt wird, sondern eine Anekdote über einen anderen, über Adorno. Die Anekdote selbst ist natürlich dokumentierbar. In den „Literaturhinweisen zu den Geistergesprächen“ hinten im Buch wird auf einen Aufsatz von Gunzelin Schmid Noerr mit dem Titel Adornos Erschaudern verwiesen, ${ }^{27}$ und in der Tat hat Adorno diese Szene in seinem kurzen Text Zweimal Chaplin in teilweise gleich-

26 Lethen: Die Staatsräte, 162-163.

27 Schmid Noerr, Gunzelin. „Adornos Erschaudern. Variationen über den Händedruck“. In: Vierzig Jahre Flaschenpost: Dialektik der Aufklärung 1947-1987. Hg. Willem van Reijen, Gunzelin Schmid Noerr. Frankfurt a. M.: Fischer 1987, 233-241. 
lautenden Worten geschildert. ${ }^{28}$ Aber sie bezieht sich, wie an der Darstellung von Gründgens selbst ablesbar, auf einen Vorfall aus dem Jahre 1946. Der Kriegsversehrte, von dem die Rede ist, hat seine beiden Hände im Zweiten Weltkrieg verloren, und davon handelt auch der genannte Film. ${ }^{29}$ Das Geistergespräch findet jedoch vor dem Zweiten Weltkrieg statt. Dass Sauerbruch im vorangegangenen Gespräch von den idealen „Experimentierbedingungen“30 für die Entwicklung von Prothesen aufgrund der vielen Versehrten nach dem Ersten Weltkrieg gesprochen hatte, verleiht dem Ganzen einen makabren Hintersinn.

Aus der Perspektive des dokufiktionalen Erzählens ergibt sich daraus die Frage, ob solche Machenschaften gegen eine Regel verstoßen. Worauf darf sich der Leser in einem dokufiktionalen Text verlassen? Ich glaube, es liegt in der Logik des ,semidokumentarischen Pakts', dass man das nur von Fall zu Fall entscheiden kann. ${ }^{31}$ In diesem Fall, würde ich sagen, ist der Anachronismus kein Regelverstoß. In gewisser Weise folgt das schon daraus, dass die Geistergespräche eben als

28 Hier die Stelle zum Vergleich: „Daß ich von ihm [Chaplin] rede, darf ich vielleicht mit einem Privileg rechtfertigen, das mir, ganz ohne mein Verdienst, zuteil wurde. Er hat mich nachgemacht; sicherlich bin ich einer der wenigen Intellektuellen, denen das widerfuhr, und die von dem Augenblick Rechenschaft zu geben vermögen. Wir waren, mit vielen anderen zusammen, in einer Villa in Malibu, am Strande außerhalb von Los Angeles, eingeladen. Einer der Gäste verabschiedete sich früher, während Chaplin neben mir stand. Ich reichte jenem, anders als Chaplin, ein wenig geistesabwesend die Hand und zuckte fast zugleich heftig zurück. Der Abschiednehmende war einer der Hauptdarsteller aus dem kurz nach dem Krieg berühmt gewordenen Film ,The Best Years of Our Life‘ [sic!]; er hatte im Krieg die Hand verloren und trug an deren Statt aus Eisen gefertigte aber praktikable Klauen. Als ich die Rechte schüttelte, und sie auch noch den Druck erwiderte, erschrak ich aufs äußerste, spürte aber sofort, daß ich das dem Verletzten um keinen Preis zeigen dürfte, und verwandelte mein Schreckgesicht im Bruchteil einer Sekunde in eine verbindliche Grimasse, die weit schrecklicher gewesen sein muß. Kaum hatte der Schauspieler sich entfernt, als Chaplin bereits die Szene nachspielte. So nah am Grauen ist alles Lachen, das er bereitet und einzig in solcher Nähe seine Legitimation gewinnt und sein Rettendes." (Adorno, Theodor W. „Zweimal Chaplin“. In: Gesammelte Schriften, Bd. 10. Kulturkritik und Gesellschaft I. Prismen. Ohne Leitbild. Hg. Rolf Tiedemann unter Mitwirkung von Gretel Adorno, Susann BuckMorss und Klaus Schultz, Frankfurt a. M.: Suhrkamp 1977, 362-366, hier 365-366.)

29 Es handelt sich um Harold Russell, der übrigens der einzige Schauspieler ist, der jemals für ein und dieselbe Rolle - eben die in diesem Film - zwei Oscars bekommen hat (einen für den besten Nebendarsteller und einen Ehren-Oscar als Symbolfigur für die Veteranen). Vgl. zur Begebenheit und ihren Hintergründen auch Michael Niehaus: „,Gegenbeispiel‘. Eine Erwiderung auf Thomas Bedorfs Überlegungen zum Händedruck als phänomenlogischem Leitbeispiel“'. In: $z . B$. Zeitschrift zum Beispiel (2020), 11-27, besonders 18-27.

30 Lethen: Die Staatsräte, 158.

31 Es geht hier um ein anderes Feld als das, was Thomas Strässle in einem Essay Fake und Fiktion. Über die Erfindung der Wahrheit, als „faketional“ (Strässle, Thomas. Fake und Fiktion. Über die Erfindung der Wahrheit. München: Carl Hanser 2019, 39-51) bezeichnet. Auch die von Sträss- 
Geistergespräche ausgewiesen sind. Anders gewendet: Das erklärtermaßen hypothetische Erzählen kann sich ebenso gut auf Dokumente aus der Vergangenheit wie aus der Zukunft beziehen, wenn sie passen. Und hier passen sie gewiss. Abgesehen davon kann jeder den Betrug bemerken.

Noch auf ein anderes dokufiktionales Erzählverfahren ist an dieser Stelle hinzuweisen. Es ist das der indirekten Wiedergabe im Modus einer größeren Distanz. Abgesehen davon, dass die Anwesenden ziemlich misslungene Kommentare zu der Adorno-Anekdote abgeben, hat Lethen (oder der Erzähler?) sie Gründgens auch ein wenig verfälscht wiedergeben lassen. ${ }^{32}$ Freilich ist das fehlerhaft formuliert, da sich der fiktive Gründgens ja gar nicht auf ein Dokument bezieht. Bei Adorno ist es eben zunächst eine Anekdote über Charlie Chaplin, von Peinlichkeit für ihn selbst ist nicht die Rede. Das allgemeine Verfahren besteht jedenfalls darin, das Dokument, auf welches Bezug genommen wird, einerseits in indirekter Rede (aus der man auf einen Wortlaut nicht schließen kann) wiederzugeben sowie andererseits durch den Filter einer weiteren Redeinstanz (deren Einsichtsfähigkeit und Wahrhaftigkeit zweifelhaft sein können). Mögen die Herren über Adornos von Gründgens überlieferten Kommentar lachen („Diese Juden!“), er hat in Wahrheit eben zwar wörtlich ähnlich, sinngemäß aber ganz anders gelautet. Umgekehrt kann man den verfälschten Titel des Films - er heißt nicht „The Best Years of Our Life“, sondern The BEst YEARs of OUR LIVES - in der Rede von Gründgens sozusagen Adorno in die Schuhe schieben; dort steht er nämlich ebenfalls falsch.

\section{3.}

Das dritte Beispiel ist in jedem Falle dem Reich der Literatur zuzurechnen, obwohl es in herkömmlicher Weise wenig ,literarisch' ist, insofern es den Geist der äußersten Reduktion atmet. Es handelt sich um das Buch Felix und Felka von Hans Joachim Schädlich aus dem Jahr 2018. Eine paratextuelle Gattungszuordnung hat das Buch nicht; ebenso wie Die Staatsräte und 1913 finden sich am Ende Hinweise auf Quellen und sogar Zitatnachweise. Auch hier kommen keine fiktiven Figuren

\footnotetext{
le angestellten Analysen bewegen sich leider, was die erzähltheoretische Seite angeht, in einer binären Logik.

32 Aber das hat Jürgen Habermas 1969 in seinem Nachruf auf Adorno auch nicht anders gemacht (vgl. Harrasser, Karin. „Subjektivierung als Intussuszeption. Mit Adorno und Chaplin auf einer Party in Malibu“. In: Szenographien des Subjekts. Hg. Lars Friedrich, Karin Harrasser, Céline Kaiser. Wiesbaden: Springer 2018, 155-166, hier 158-159).
} 
vor: Felix und Felka, das ist das jüdische Malerehepaar Felix Nussbaum und Felka Platek, beide 1944 in Auschwitz ermordet. Die Kraft dieser Dokufiktion beruht darauf, dass sie ohne jeden doppelten Boden auskommt.

Felix Nussbaum stammte aus einer wohlhabenden Familie in Osnabrück (wo ihm jetzt ein von Daniel Libeskind entworfenes und gebautes Museum gewidmet ist). Das chronologisch aufgebaute Buch beginnt 1933 mit der Entfernung Nussbaums aus der Villa Massimo in Rom. Es folgt eine lange Reihe von Stationen vorübergehender Wohnsitze, des Untertauchens, der Internierung, der Flucht, auf denen Felix Nussbaum (dessen zuvor entstandene Werke 1932 bei einem Brand in Berlin fast alle zerstört worden waren) den Großteil seines erhaltenen Euvres schuf. Das Buch schreitet diese Reihe ab in einzelnen, unverbundenen Szenen, bisweilen unterbrochen durch eingerückte Briefe Nussbaums, und zwar gewissermaßen unter Verzicht auf eine Erzählinstanz. Hier ein Beispiel:

Felix und Felka werden am 6. Oktober 1937, 11.00 Uhr, im Rathaus des I. Brüsseler Bezirks gesetzlich getraut.

Nach der der Trauung gehen sie in ein Restaurant zum Mittagessen.

„Schade, daß wir jetzt keine jüdische Hochzeit feiern“, sagt Felka. „Du trägst einen weißen Kittel, ich bin verschleiert.“

„Woher soll ich einen weißen Kittel nehmen.“

„Wir werden von unseren Eltern unter die seidene Chuppe geführt.

Der Rabbi hält einen Becher Wein in der Hand und spricht den Segen.

Wir trinken jeder einen Schluck.

Du steckst mir vor den beiden Zeugen einen silbernen Ring an den Zeigefinger und sagst:

,Durch diesen Ring bist zu mir angelobt nach dem Gesetz des Mose und Israels.““

„Und wir hatten heute nicht einmal Eheringe.“

„Der Rabbi liest den Ehevertrag vor.

Er gibt ihn dir und du überreichst ihn mir.

So ist mein Leben gesichert.“

„Eigentlich sicherst du mein Leben“, sagt Felix. ${ }^{33}$

Der Dialog setzt sich noch eine Weile fort, wobei deutlich wird, dass der unterschiedliche Stellenwert, den das Paar der jüdischen Hochzeitszeremonie beimisst, damit zusammenhängt, dass Felix aus einer assimilierten jüdischen Familie stammt, Felka hingegen aus einer armen ostjüdischen Familie.

Der erste Satz der zitierten Passage (die, wie das ganze Buch, wiederum im Präsens steht) ist keine Fiktion, sondern gibt nur ein dokumentiertes Faktum wieder. Die im zweiten Satz enthaltene Proposition ließ sich vermutlich einem Brief entnehmen. Es wird nicht gesagt, in welchem Restaurant das Essen stattfindet, wie das Ambiente ist, was die beiden gegessen haben usw. Das Restaurant (das

33 Schädlich, Hans Joachim. Felix und Felka. Reinbek b. Hamburg: Rowohlt 2018, 64. 
wir uns als Leser eben nicht ,ausmalen' sollen) ist also nur der formale Schauplatz des Dialogs. Fiktion ist folglich nur der Dialog selbst, der als solcher schon deshalb, weil nur die beiden Dialogpartner anwesend ist, nicht dokumentiert worden sein kann, aber gleichwohl mit den bei Lethen vermiedenen Anführungszeichen in direkter Rede eingerückt ist. Insofern wird zwar einerseits mit Nachdruck behauptet, dass genau dies wirklich gesagt worden ist, andererseits geht zugleich das Wissen, dass gewiss nicht so gesprochen wurde, in die Rezeptionssituation ein. Denn so spricht man eben nicht: so hölzern, so formalisiert, so verfremdet.

Hinzu kommt im zitierten Abschnitt, dass die ausgefallene Beschreibung der Trauung in Brüssel ersetzt wird durch die Beschreibung der Trauung, wie sie in der polnischen Heimat Felkas hätte stattfinden können. Eine solche Trauung lässt sich beschreiben, weil sie sich als ein ritualisierter Vorgang wiederholt. Wenn diese Beschreibung - im Grunde eine iterative Erzählung - Felka als einem der Dialogpartner in den Mund gelegt wird, so wird damit behauptet, dass die formlose Trauung vor dem Brüsseler Standesamt von ihr auf der Folie der traditionellen Trauung aufgefasst wird.

Wie lässt sich der Status dieser Dialogpassagen, die den weitaus überwiegenden Anteil des Buches ausmachen, bestimmen? Man kann sagen, dass sie dazu da sind, eine Wirklichkeit zu dokumentieren, ohne sie abzubilden. Es handelt sich gewissermaßen um Protokollsätze. Und Protokolle sind Dokumente, die nicht das enthalten, was tatsächlich gesagt worden ist, sondern was die Beteiligten gesagt zu haben unterschreiben können. ${ }^{34}$ In diesem Sinne stellen viele der Dialoge in diesem Buch - es sind übrigens ausschließlich Dialoge zwischen Zweien, meist zwischen Felix und Felka - nicht nur ihre Künstlichkeit aus, sondern sind gleichsam als hypothetische Verdichtungen (oder als Figurationen) dessen zu verstehen, was die Beteiligten als Zusammenfassung ihrer Situationsbeschreibung gelten lassen könnten. Der Erzähler wird zum Protokollführer und das Fiktionale wird seiner Logik nach dokumentarisch, ohne zu fingieren, ein Dokument zu sein.

\footnotetext{
34 Nur der direkte Stil, die wörtliche Rede, heißt es z. B. in einem Lehrbuch für Untersuchungsrichter aus dem 19. Jahrhundert, biete die Gewähr dafür, dass derjenige, der „das Protokoll später liest, sich lebendig in die Seele und damalige Stimmung des Deponenten zurückversetzen kann.“ (Jagemann, Ludwig Hugo Franz von. Handbuch der gerichtlichen Untersuchungskunde, Bd. 1. Frankfurt a. M.: Kettembeil 1838, 615) Der direkte Stil erweckt den Anschein einer Unmittelbarkeit, die gerade nicht gegeben ist. Die Unmittelbarkeit ist eine Fiktion, das Protokoll eine Konstruktionsleistung. In demselben Lehrbuch wird daher erklärt, „nur ein Laie [...] könnte auf die Meinung verfallen, die Reden und Gegenreden, Vorträge und Erzählungen seien wörtlich so aufeinandergefolgt, wie sie in den Acten stehen“; in Wahrheit sei jeder „Satz, der in die Akten geschrieben wird, [...] das Ergebnis einer Unterredung“ (610-611).
} 
Das letzte Beispiel ist hinsichtlich der Erzählverfahren und Erzähltechniken das komplexeste: das Buch Verzeichnis einiger Verluste von Judith Schalansky, ebenfalls aus dem Jahr 2018. Es handelt sich im emphatischen Sinne um ein Buch, d. h. um ein Buch ohne Zuordnung zu einer Gattung, wenn man nicht den Begriff „Verzeichnis“ als eine (dann nichtfiktionale) Textsortenbezeichnung gelten lassen will. ${ }^{35}$ Nebst einer kurzen Vorbemerkung und einem Vorwort-Essay über Verlust und Gedächtnis besteht das Buch aus zwölf Abschnitten oder Texten, die mit jeweils genau sechzehn Seiten alle den gleichen Umfang haben, aber in Stil und Genre stark divergieren. Es gibt ganz und gar fiktionale Texte (in denen auch fiktive Figuren auftreten), aber auch Formen des nature writing, zum Autofiktionalen tendierende Texte sowie solche, die dem Feld des Dokufiktionalen zuzurechnen sind. So gleich der erste Text, der mit „Südliche Cookinseln TUANAKI, auch Tuanabe“36 überschrieben ist. Nach einer kurzen, typographisch abgesetzten Vorinformation ${ }^{37}$ über die besagte Insel, die anscheinend Ende 1842 bei einem Seebeben untergegangen ist, beginnt der Text mit dem Satz:

\footnotetext{
Es war an einem lichten und vollkommen windstillen Apriltag vor genau sieben Jahren, als ich eine mir unbekannte Insel namens Ganges auf einem Globus der Kartenabteilung der Staatsbibliothek entdeckte. ${ }^{38}$
}

An diesem Satz sieht man schon, was den Komplexitätsgrad dokufiktionalen Erzählens entscheidend erhöht: Es ist der unklare Status der ersten Person Singular, die zudem das Präteritum nach sich zieht. ${ }^{39}$ In den anderen drei Beispielen kam ein auf die Erzählinstanz zu beziehendes „Ich“ entweder gar nicht vor oder lediglich im definierten paratextuellen Rahmen eines Metadiskurses. Hier aber ist mit der Anwesenheit dieses Pronomens sogleich die schillernde autofiktionale Dimension auf dem Plan.

Indes spielt der autofiktionale Aspekt zunächst einmal eine untergeordnete Rolle, da dieses „Ich“ hier (anders als in anderen Texten im Verzeichnis einiger Verluste) nicht mit einer Biografie versehen wird, sondern ausschließlich als Ver-

35 Die Verfasserin ist auch als Buchgestalterin tätig und erfolgreich.

36 Schalansky, Judith. Verzeichnis einiger Verluste. Berlin: Suhrkamp 2018, 29.

37 Alle sechzehn Texte setzen mit einer solchen Vorinformation ein.

38 Schalansky: Verzeichnis einiger Verluste, 29.

39 Natürlich gibt es in der Moderne eine Unmenge von Erzähltexten in der ersten Person Präsens. Die - hier nicht näher zu erläuternde - Behauptung besteht darin, dass dies keine dokufiktionalen Texte sein können. 
mittlungsinstanz dient: Es ist ein (figuratives) Ich, das durch eine Suchbewegung (also durch Fremdreferenz) charakterisiert ist. Und auf dieser Suche (nach Dokumenten) kommen Variationen verschiedener Modi des dokufiktionalen Erzählens zum Einsatz, die in den drei anderen Beispielen bereits zu besichtigen waren. Das „Ich“ nähert sich der verlorenen, als utopischem Ort vorgestellten Insel Tuanaki zunächst über die zugänglichen Dokumente - insbesondere jener, die mit der Expedition des Namensgebers der im Untertitel genannten Inseln in Zusammenhang stehen, also James Cook:

Es bestand kein Zweifel: Jener Entdecker, den ein kleiner Kontinent bis in die heutige Zeit als den größten unter all seinen in jeden Winkel der Welt vordringenden Seefahrern preist, musste auf seiner dritten und letzten Fahrt Tuanaki nur knapp verfehlt haben, ja, seine beiden einst als Kohlenfrachter im Nebel von Whitby vom Stapel gelaufenen Schiffe im Laufe des 27. März 1777 nur unweit ihres Gesichtskreises vorübergesegelt sein - mit geblähtem Tuch, stolz wie Fregatten, in vollem Ornat. ${ }^{40}$

Ganz anders als in Felix und Felka treibt das hier aufgetauchte Ich einen beträchtlichen sprachlichen Aufwand, um die Dokumente in ein eigenes Vorstellungsbild zu verwandeln, deren Ausgangspunkt das Modalverb „musste“ ist, welches wiederum auf das konjekturale Erzählen als dokufiktionales Verfahren verweist. Freilich ist alles, was in der Folge über die Reise der Resolution und der Discovery erzählt wird, von Quellen gedeckt, auch wenn es eine distanziert vorgestellte, mit iterativen Bestandteilen durchsetzte und mit Reflexionen versehene Version derselben ist. Das Land, das Cook und seine Leute zwei Tage später entdecken, ist nicht Tuanaki, sondern das etwa hundert Seemeilen entfernte Mangaia, wo ein Insulaner schließlich an Bord der Resolution kommt. Eingeleitet von Wendungen wie „So viel immerhin ist sicher [...]“41 und „Verbürgt ist auch [...]“42 nähert sich die Erzählung dann der Begegnung Cooks mit dem Insulaner Mourua:

Eine Zeitlang musste er dem Kapitän in dessen Kajüte gegenübergestanden haben, Aug in Aug, einander musternd, wie zwei Tiere, die sich nie zuvor gesehen hatten: Zwei Männer, der gleichmäßig runde Schädel Mouruas neben dem an einen Vogel erinnernden Cooks [...].43

Die Begegnung zwischen den Beiden ist zugleich verbürgt und fantasiert. Ein solcher Darstellungsmodus, der in anderer Form auch im historiographischen Erzählen vorkommt, kann weder als fiktional noch als faktual beschrieben wer-

40 Schalansky: Verzeichnis einiger Verluste, 32.

41 Schalansky: Verzeichnis einiger Verluste, 37.

42 Schalansky: Verzeichnis einiger Verluste, 37.

43 Schalansky: Verzeichnis einiger Verluste, 37-38. 
den. ${ }^{44}$ Weil ein Verweilen der Schiffe vor Mangaia wegen der Brandung als unmöglich erachtet wird, bleibt es bei dieser kurzen Begegnung. Lediglich eine „eiserne Axt “45 wechselt den Besitzer und wird zum Anhaltspunkt für die dokufiktionale Ausweitung. Der nächste Absatz beginnt mit der Wendung „Und hier verließen mich die Überlieferungen der Zeugen“46. Die Ich-Erzählerin (etliche Erzähltheoretiker`innen müssten die Rechtmäßigkeit der weiblichen Form bezweifeln, da das Geschlecht dieses „Ich“ nirgends indiziert wird) möchte das unbetretene Land in der Vorstellung betreten und kann sich dafür durchaus auf spätere „Quellen“47 stützen, ${ }^{48}$ aber wie sich die Szene nach der Rückkehr Mouruas auf Magaia fortsetzen lässt, lässt sich daraus nicht erschließen:

\begin{abstract}
So konnte ich nur mutmaßen, wie Mourua am Ufer empfangen wurde, auch wenn ich aus irgendeinem unlauteren Grund mir genau ausmalte, wie ihn seine Landsleute mit Fragen um das Wesen und die Herkunft der bleichen Besucher bedrängten und einhellig zu dem Schluss kamen, Tangaroa hätte sie geschickt, jener einst auf Mangaia angebetete Gott, der vor Urzeiten im Kampf seinem Bruder Rongo unterlegen und aufs offene Meer geflohen war. Und ich sah vor mir [...]. In meiner kümmerlichen Phantasie war es Mourua, der als erster vor das Idol trat $[. .]$.
\end{abstract}

Hier wird erzählt, indem gesagt wird, dass das Erzählte nicht im einfachen Indikativ steht. Dieses Zeigen des Nicht-Indikativischen im Erzählvorgang ist nur möglich, weil dieser auf ein Aussagesubjekt bezogen werden kann, von welchem es in 1913 von Florian Illies nur eine Spur gibt, das hier aber als ein „Ich“ auftritt, welches sich dem Begehren nach dem Dokumentierbaren ganz überantwortet hat. Im Schlussakkord des Textes stößt das Ich tatsächlich auf den Bericht eines polynesi-

44 Vgl. etwa die exemplarische Einzelanalyse, die Stephan Jaeger in seinem Beitrag Erzählen im historiographischen Diskurs zu den Erzählverfahren in Jörg Friedrichs Buch Der Brand. Deutschland im Bombenkrieg 1940-1945 (Friedrich, Jörg. Der Brand. Deutschland im Bombenkrieg 19401945. München: List 2002) vornimmt. Jaeger stellt abschließend fest: „Es zeigt sich, dass die dualistische Unterscheidung von Historie und Fiktion die Besonderheit von Friedrichs Text nicht erklären kann. Es ist ohne Zweifel Geschichte, aber Geschichte wird nicht im weitesten Sinne nachgeahmt oder referiert, sondern als Konstrukt - als Geschichtswahrnehmung - re-inszeniert, also neu erschrieben.“ (Jaeger, Stephan. „Erzählen im historiographischen Diskurs“. In: Wirklichkeitserzählungen. Felder, Formen und Funktionen nicht-literarischen Erzählens. Hg. Christian Klein, Matías Martínez. Stuttgart: J. B. Metzler 2009, 110-135, hier 131).

45 Schalansky: Verzeichnis einiger Verluste, 38.

46 Schalansky: Verzeichnis einiger Verluste, 39.

47 Schalansky: Verzeichnis einiger Verluste, 39.

48 Dem Verzeichnis einiger Verluste ist anders als den drei übrigen Büchern keine Bibliografie angehängt. Vermutlich ist hier vor allem das Mangaia gewidmete Buch From Darkness to Light in Polynesia von Rev. William Wyatt Gill aus dem Jahr 1894 gemeint.

49 Schalansky: Verzeichnis einiger Verluste, 39-40. 
schen Matrosen, der im Auftrag des Kapitäns eines englischen Schiffes 1842 sechs Tage bei den Insulanern auf Tuanaki verbracht hat. An diesem Punkt springt ihre Darstellung in die Präsentation einer Szene um. In einer finalen Verschmelzung von Dokument und Fiktion wird die Szene der Begegnung wie folgt geschildert:

Einer von ihnen, zweifellos der Älteste, bedeutete dem Besucher, einzutreten, und als dieser der Einladung folgte, fragte der Alte nach dem Kapitän des Schiffes.

„Er ist an Bord“, antwortete der Matrose wahrheitsgemäß.

„Warum kommt er nicht an Land?“, fragte der Mann, ohne die Miene zu verziehen. Um seinen Hals baumelte das Muschelhorn.

„Er hat Angst, ihr könntet ihn töten.“

Stille kam auf, und für einen kurzen Moment schien die Brandung gefährlich nah. Der Alte sah ins Blätterwerk des Waldes. Endlich sagte er vollkommen ruhig: „Wir wissen nicht, wie man tötet. Wir wissen nur, wie man tanzt." ${ }^{00}$

Kann es sein, dass das keine Fiktion ist, dass dieser Satz, demzufolge es einen auf immer untergegangenen Sehnsuchtsort des ewigen Friedens gibt, wo man nicht zu töten, sondern nur zu tanzen weiß, tatsächlich dokumentiert ist? Hier der Bericht, den Judith Schalansky gelesen hat:

The chief asked me, ,Whence do you come? From Araura'? I replied ,Yes!' ,Come inside the house!' So I went inside; there were non but men there, no women as they have a separate house. After I had sat down, the chief asked again, ,Do you come from Araura,' to which I replied, ,I came from Araura“ [...]. ,A! Where is the captain of your ship?‘ I told him he was with the boat. ,He is afraid, lest you should kill him!‘ ,We do not kill men; we only know how to dance (ura) and sing; we know nothing of war. ${ }^{\text {51 }}$

Der Ich-Erzählerin, also Judith Schalansky, gibt ihre Lektüre den abschließenden Gedanken ein, dass „der Mythos [...] die höchste aller Wirklichkeiten“ ist und „die Bibliothek der wahre Schauplatz des Weltgeschehens“. 52

50 Schalansky: Verzeichnis einiger Verluste, 43.

51 Gill, Rev. William Wyatt/Smith, Stephenson Percy. „Tuanaki, the lost Island“. In: Rarotonga Records: Being Extracts from the Papers of the Late Rev. W. Wyatt Gill. Hg. The Polynesian Society, 1916, 29-31, hier 30-31. Dieser drei Seiten umfassende, in dem Buch Rarotonga Records: Being Extracts from the Papers of the Late Rev. W. Wyatt Gill der Polynesian Society abgedruckte Bericht, der diese Szene enthält, ist wohl alles, was von der Insel Tuanaki übrig geblieben ist.

52 Schalansky: Verzeichnis einiger Verluste, 44. 


\section{5.}

Welche vorläufigen Schlüsse über die Modi des dokufiktionalen Erzählens lassen sich daraus ableiten? Der Modus ist bekanntlich eine der drei Kategorien, unter denen Gérard Genette den narrativen Diskurs betrachtet und geordnet hat. Die beiden anderen sind Zeit und Stimme. Die Begründung, die Genette zu Beginn seines Discours du récit für diese Dreiteilung angeboten hat, sind jedoch in den anschließenden Diskussionen mehr oder weniger unbeachtet geblieben. Er will nämlich „die Probleme der Analyse des narrativen Diskurses nach Kategorien [...] ordnen, die der Grammatik des Verbs entlehnt werden“53. Klar ist: Das Verb steht in einem Tempus (das ist die Kategorie der Zeit) und es steht in einer bestimmten Person (das ist die Kategorie der Stimme). Aber wie verhält es sich mit dem Modus?

Die Erzählung hat ja nicht die Funktion, einen Befehl zu erteilen, einen Wunsch zu formulieren, eine Bedingung anzugeben usw., sondern bloß die, eine Geschichte zu erzählen, d. h. über (wirkliche oder fiktive) Tatsachen zu „berichten“, sodass ihr einziger, jedenfalls charakteristischer Modus streng genommen nur der Indikativ sein kann. ${ }^{54}$

Wenn Genette in der Folge Perspektive und Distanz als die „beiden wesentlichen Weisen jener Regulierung der narrativen Information“ bezeichnet, „die der Modus darstellt“, so versucht er das nicht zuletzt damit zu rechtfertigen, dass es nicht nur „einen Unterschied zwischen behaupten, befehlen, wünschen usw. gibt, sondern auch graduelle Unterschiede in der Behauptung, und dass diese Unterschiede ihren Ausdruck für gewöhnlich in modalen Variationen finden“. ${ }^{55}$ Man könne das, was man erzählt, „mehr oder weniger nachdrücklich“ erzählen (aus geringerer oder größerer Distanz), und man könne es „unter diesem oder jenem Blickwinkel“ erzählen. ${ }^{56}$

Ich meine, dass dieser Ansatz für eine nähere Bestimmung der Modi des dokufiktionalen Erzählens fruchtbar gemacht werden kann, wenn man die bei Genette vorausgesetzte Opposition wirklich/fiktiv (bzw. faktual/fiktional) relativiert. Betrachtet man die Modi, von denen in der Grammatik gesprochen wird, so geht es dort nicht nur um ,Grade der Behauptung' (obwohl auch das schon bemerkens-

53 Genette, Gérard. Die Erzählung. 3. Auflage. Paderborn: Fink 2010, 14.

54 Genette: Die Erzählung, 103.

55 Genette: Die Erzählung, 103. (Herv. i. O.)

56 Genette: Die Erzählung, 103. (Herv. i. O.) 
wert ist). ${ }^{57}$ Man unterscheidet neben dem Indikativ (also der Behauptung, dass etwas so ist), dem Energikus (der kategorischen Behauptung, dass es so ist) sowie dem Imperativ (dass etwas so sein soll) und dem Optativ (dass es so sein möge) auch noch den Nezessativ (dass etwas so sein muss), Potentialis (dass es so sein kann) und Konditionalis (dass es unter einer bestimmten Voraussetzung so ist). Insbesondere den drei letzten Modi - also Nezessativ, Potentialis und Konditionalis scheinen mir die in den vorliegenden Überlegungen skizzierten dokufiktionalen Erzählverfahren und Erzähltechniken zu entsprechen, die es genauer zu bestimmen und zu analysieren gilt.

\section{Literatur}

Adorno, Theodor W. „Zweimal Chaplin“. In: Gesammelte Schriften, Bd. 10. Kulturkritik und Gesellschaft I. Prismen. Ohne Leitbild. Hg. Rolf Tiedemann unter Mitwirkung von Gretel Adorno, Susann Buck-Morss und Klaus Schultz. Frankfurt a. M.: Suhrkamp 1977, 362-366.

Avanessian, Armen/Hennig, Anke (Hg.). Der Präsensroman. Berlin/New York: De Gruyter 2013. Bauschinger, Sigrid. Else Lasker-Schüler. Eine Biographie. Göttingen: Wallstein 2004.

Bidmon, Agnes. „Streng vertraulich! Dokufiktionales Erzählen als Schreibweise des Politischen in der Literatur der Gegenwart anhand von Ilija Trojanows Macht und Widerstand“. In: Politische Literatur. Begriffe, Debatten, Aktualität. Hg. Christine Lubkoll, Manuel Illi, Anna Hampel. Stuttgart: J.B. Metzler 2019, 421-440.

Friedrich, Jörg. Der Brand. Deutschland im Bombenkrieg 1940-1945. München: List 2002.

Genette, Gérard. Die Erzählung. 3. Auflage. Paderborn: Fink 2010.

Gill, Rev. William Wyatt. From Darkness to Light in Polynesia. London 1894.

Gill, Rev. William Wyatt/Smith, Stephenson Percy. „Tuanaki, the lost Island“. In: Rarotonga Records: Being Extracts from the Papers of the Late Rev. W. Wyatt Gill. Hg. The Polynesian Society, 1916, 29-31.

Gittel, Benjamin. „,Wirklichkeitsverlust', ,Wirklichkeitshunger‘ und ,Neuer Realismus‘. Zur Verschränkung von Gegenwartsdiagnostik, Poetologie und Literaturwissenschaft“. In: IASL 43/1 (2018), 68-89.

57 Die gesamte Diskussion um den sprechakttheoretischen Status des Fiktionalen im Anschluss an John R. Searle dreht sich um die Kategorie der (vorgeblichen, simulierten) Behauptung (vgl. etwa zusammenfassend Onea, Edgar. „Fiktionalität und Sprechakte“. In: Fiktionalität. Ein interdisziplinäres Handbuch. Hg. Tobias Klauk, Tilmann Köppe. Berlin/Boston: De Gruyter 2014, 68-96). Solange man daran festhält, kommt man nicht zu einer adäquaten Beschreibung dokufiktionalen Erzählens. Die Notwendigkeit, auch andere Modi zu berücksichtigen, zeigt sich auch - allerdings auf Textsorten bezogen - in der zusätzlichen Unterscheidung von normativen und voraussagenden „Wirklichkeitserzählungen“ bei Christian Klein und Matías Martínez (Klein/Martínez: Wirklichkeitserzählungen, 6). 
Harrasser, Karin. „Subjektivierung als Intussuszeption. Mit Adorno und Chaplin auf einer Party in Malibu“. In: Szenographien des Subjekts. Hg. Lars Friedrich, Karin Harrasser, Céline Kaiser. Wiesbaden: Springer 2018, 155-166.

Illies, Florian. 1913. Der Sommer des Jahrhunderts. Frankfurt a. M.: Fischer 2014.

Jaeger, Stephan. „Erzählen im historiographischen Diskurs“. In: Wirklichkeitserzählungen. Felder, Formen und Funktionen nicht-literarischen Erzählens. Hg. Christian Klein, Matías Martínez. Stuttgart: J.B. Metzler 2009, 110-135.

Jagemann, Ludwig Hugo Franz von. Handbuch der gerichtlichen Untersuchungskunde. Bd. 1. Frankfurt a. M.: Kettembeil 1838.

Klein, Christian/Martínez, Matías. „Wirklichkeitserzählungen. Felder, Formen und Funktionen nicht-literarischen Erzählens“. In: Wirklichkeitserzählungen. Felder, Formen und Funktionen nicht-literarischen Erzählens. Hg. Christian Klein, Matías Martínez. Stuttgart: J.B. Metzler 2009, 1-13.

Lethen, Helmut. Die Staatsräte. Elite im Dritten Reich: Gründgens, Furtwängler, Sauerbruch, Schmitt. Berlin: Rowohlt 2018.

Martínez, Matías. „Erzählen im Journalismus“. In: Wirklichkeitserzählungen. Felder, Formen und Funktionen nicht-literarischen Erzählens. Hg. Christian Klein, Matías Martínez. Stuttgart: J.B. Metzler 2009, 179-191.

Mecke, Jochen. „Du musst dran glauben. Von der Literatur der Lüge zur Lüge der Literatur“. In: Diegesis 4/1 (2015). https://www.diegesis.uni-wuppertal.de/index.php/diegesis/article/ view/188/255 (28.10.2019).

Niehaus, Michael. „,Gegenbeispiel'. Eine Erwiderung auf Thomas Bedorfs Überlegungen zum Händedruck als phänomenlogischem Leitbeispiel. In: z.B. Zeitschrift zum Beispiel (2020), 11-27.

Onea, Edgar. „Fiktionalität und Sprechakte“. In: Fiktionalität. Ein interdisziplinäres Handbuch. Hg. Tobias Klauk, Tilmann Köppe. Berlin/Boston: De Gruyter 2014, 68-96.

Schalansky, Judith. Verzeichnis einiger Verluste. Berlin: Suhrkamp 2018.

Schädlich, Hans Joachim. Felix und Felka. Reinbek b. Hamburg: Rowohlt 2018.

Schmid Noerr, Gunzelin. „Adornos Erschaudern. Variationen über den Händedruck“. In: Vierzig Jahre Flaschenpost: Dialektik der Aufklärung 1947-1987. Hg. Willem van Reijen, Gunzelin Schmid Noerr. Frankfurt a. M.: Fischer 1987, 233-241.

Sebald, W. G. Schwindel. Gefühle. Frankfurt a. M.: Fischer 1994.

Strässle, Thomas. Fake und Fiktion. Über die Erfindung der Wahrheit. München: Carl Hanser 2019.

Wackwitz, Stephan. Ein unsichtbares Land. Familienroman. Frankfurt a. M.: Fischer 2005. Weinrich, Harald. Tempus. Besprochene und erzählte Welt. München: C.H.Beck 2001 [1964]. Zipfel, Frank. „Fiktionssignale“. In: Fiktionalität. Ein interdisziplinäres Handbuch. Hg. Tobias Klauk, Tilmann Köppe. Berlin/Boston: De Gruyter 2014, 97-124. 
